\title{
Bone marrow mesenchymal stem cell donors with a high body mass index display elevated endoplasmic reticulum stress and are functionally impaired
}

\author{
Baris Ulum ${ }^{1}$ | Hikmet Taner Teker ${ }^{1}$ | Aysun Sarikaya ${ }^{1}$ | Gunay Balta ${ }^{1,2}$ | \\ Baris Kuskonmaz $^{2}$ | Duygu Uckan-Cetinkaya ${ }^{1,2}$ | Fatima Aerts-Kaya ${ }^{1}$ (1)
}

${ }^{1}$ Department of Stem Cell Sciences, Graduate School of Health Sciences, Center for Stem Cell Research and Development, Hacettepe University, Ankara, Turkey

${ }^{2}$ Department of Pediatrics, Faculty of Medicine, Division of Hematology, Hacettepe University, Ankara, Turkey

\section{Correspondence}

Fatima Aerts-Kaya M.D., Ph.D., Department of Stem Cell Sciences, Graduate School of Health Sciences, Center for Stem Cell Research and Development (PEDI-STEM), Hacettepe University, 06100 Sihhiye, Ankara, Turkey. Email: fatimaaerts@yahoo.com

Funding information FP7 Health, Grant/Award Number: CELL-PID 261387; Turkish Society of Hematology, Grant/Award Number: 2015/3
Bone marrow mesenchymal stem cells (BM-MSCs) are promising candidates for regenerative medicine purposes. The effect of obesity on the function of BM-MSCs is currently unknown. Here, we assessed how obesity affects the function of BM-MSCs and the role of endoplasmic reticulum (ER) stress and the unfolded protein response (UPR) therein. BM-MSCs were obtained from healthy donors with a normal $(<25)$ or high $(>30)$ body mass index (BMI). High-BMI BM-MSCs displayed severely impaired osteogenic and diminished adipogenic differentiation, decreased proliferation rates, increased senescence, and elevated expression of ER stress-related genes ATF4 and CHOP. Suppression of ER stress using tauroursodeoxycholic acid (TUDCA) and 4-phenylbutyrate (4-PBA) resulted in partial recovery of osteogenic differentiation capacity, with a significant increase in the expression of ALPL and improvement in the UPR. These data indicate that $\mathrm{BMI}$ is important during the selection of BM-MSC donors for regenerative medicine purposes and that application of high-BMI BM-MSCs with TUDCA or 4-PBA may improve stem cell function. However, whether this improvement can be translated into an in vivo clinical advantage remains to be assessed.

\section{KEYWORDS}

4-phenylbutyrate, body mass index, bone marrow mesenchymal stem cells, endoplasmic reticulum stress, tauroursodeoxycholic acid

\section{1 | INTRODUCTION}

Multipotent mesenchymal stem cells (MSCs) can be easily obtained from bone marrow (BM) and adipose tissue (Malgieri, Kantzari, Patrizi, \& Gambardella, 2010; Odabas, Elcin, \& Elcin, 2014) and have rapidly gained importance for their use in regenerative medicine (Nombela-Arrieta, Ritz, \& Silberstein, 2011). Maintenance of differentiation capacity and stemness of MSCs is crucial for their clinical application. With steadily increasing rates of obesity (body mass index $[\mathrm{BMI}] \geq 30 \mathrm{~kg} / \mathrm{m}^{2}$; Turconi $\&$ Hellas, 2007; WHO, 2000), the elucidation of the effects of obesity on stem cell functions has become equally more important. Obesity negatively affects the function of multiple stem cell types, including hematopoietic stem cells, adipose tissue derived stromal cells (ADSCs), skeletal muscle stem cells, and even germ cells (Ambrosi et al., 2017; Badimon \& Cubedo, 2017; Baptista et al., 2009; de Girolamo, Lucarelli, et al., 2013; De Girolamo, Stanco, et al., 2013; Emmons, Niemiro, \& De Lisio, 2017; Malgieri et al., 2010; Mihaylova, Sabatini, \& Yilmaz, 2014), and has a negative impact on the differentiation of BM-MSCs in mice (C. L. Wu, Diekman, Jain, \& Guilak, 2013). Differentiation of ADSCs of overweight humans is skewed toward the adipogenic lineage (Baptista et al., 2009) and the life span of ADSCs is significantly reduced in obese humans, as evident from early senescence and loss of differentiation (de Girolamo, Lucarelli, et al., 2013; De Girolamo, Stanco, et al., 2013). Although ADSCs and BM-MSCs share many biological features, 
significant differences have been found in terms of immunophenotypic, differentiation potentials, transcryptomic analyzes, proteomics, and immunomodulatory properties (Strioga, Viswanathan, Darinskas, Slaby, \& Michalek, 2012). The effect of obesity on the function of BM-MSCs is unknown, although an increase in BM adipocytes has been shown to negatively affect the BM niche (Adler, Kaushansky, \& Rubin, 2014; Emmons et al., 2017), disturb cell-cell interactions, hematopoiesis, and bone regeneration (Ambrosi et al., 2017). Therefore, it is conceivable that BM-MSCs, similar to ADSCs, may lose their differentiation capacities and stemness as a result of obesity. This may have direct implications for the use of MSCs obtained from high-BMI donors in regenerative medicine.

Accumulation of mis- or unfolded proteins in the lumen of the endoplasmic reticulum (ER) results in ER stress and initiates a self-rescue process, known as the unfolded protein response (UPR; Bravo et al., 2013). Under physiological conditions, the stress sensors inositolrequiring enzyme-1 $\alpha$ (IRE1 $\alpha$ ), protein kinase RNA-like endoplasmic reticulum kinase (PERK), and activating transcription factor 6 (ATF6) are linked to binding immunoglobulin protein $(\mathrm{BiP}) / 78-\mathrm{kDa}$ glucoseregulated protein (GRP78), a small chaperone molecule (J. Wu \& Kaufman, 2006). During ER stress, BiP/GRP78 becomes separated from the sensors, IRE1 $\alpha$ and PERK undergo transphosphorylation, ATF6 is transported to Golgi and cleaved, and various transcription factors (XBP1, ATF4, ATF6, NFKB, CHOP) and protein kinases (JNK, AKT) are stimulated, resulting in the activation of the UPR (Park \& Ozcan, 2013) to restore homeostasis (Bravo et al., 2013). Each of these pathways yields a specialized response during differentiation and metabolic processes (J. Wu \& Kaufman, 2006). Tauroursodeoxycholic acid (TUDCA) attenuates ER stress and prevents UPR dysfunction, and has been shown to regulate stem cell differentiation into adipogenic and osteogenic lineages (Cha et al., 2016, 2014). Its main action is channeled through the PERK pathway, modulating ATF4 and CHOP expression (Malo et al., 2010). Sodium 4-phenylbutyrate (4-PBA) stabilizes protein conformation in the ER and represses UPR activation, and has been shown to inhibit adipogenesis by modulation of the UPR (Basseri, Lhotak, Sharma, \& Austin, 2009). The effects of 4-PBA are mediated through the modulation of both PERK and IRE1 $\alpha$ pathways (Kim et al., 2012).

In this study, we functionally compared BM-MSCs from high-BMI (>30) and normal-BMI (<25) donors, and assessed the levels of ER stress during expansion and differentiation. In addition, we investigated how suppression of ER stress, using TUDCA and 4-PBA, affected expansion and differentiation capacities of BM-MSCs from high-BMI donors. We hypothesized that the modulation of ER stress in high-BMI BM-MSCs could be used to produce better quality cellular products for regenerative medicine applications.

\section{2 | MATERIALS AND METHODS}

\section{1 | Isolation and expansion of human BM-MSCs}

Use of small samples $(3-5 \mathrm{ml})$ of BM collected for transplantation purposes was approved by the Hacettepe University Ethical Committee (GO-15/130-30) and written informed consent was obtained from each BM donor. Donors were divided into two groups based on their
BMI: normal BMI (BMI <25, 17.62 \pm 4.27 , age 8-36, $n=8$ ) and high $\mathrm{BMI}(\mathrm{BMI}>30,30.85 \pm 4.16$, age $14-58, n=9)$. MSCs were cultured in complete medium (DMF10), consisting of 60\% DMEM-LG (31885-049; Life Technologies, UK) and 40\% MCDB-201 (\#M6770-1L; Sigma, Saint Louis, MO), supplemented with $10 \%$ fetal bovine serum (FBS; 10270 106, lot \#41F1133K; Gibco, UK) , 1\% penicillin/streptomycine (A2213; Biochrom AG, Germany), and $2 \mathrm{mM}$ L-glutamine (Biochrom AG). Cultures were maintained at $37^{\circ} \mathrm{C}$ and $5 \% \mathrm{CO}_{2}$, and medium was replaced every 3-4 days. Experiments were carried out with BMMSCs at passage 3 .

\section{2 | Immunophenotypic characterization of BM-MSCs}

MSCs were stained with $5 \mathrm{ul}$ of antibodies against CD90 (555596, clone 5E10; Becton Dickinson, San Jose, CA), CD73 (550257, clone AD2; Becton Dickinson, San Jose, CA), CD44 (17-0441-82, eBiosciences, San Diego, CA), CD105 (12-1057-41, clone SN6; eBioscience, San Diego, CA), HLA-DR (347403, clone L243; Becton Dickinson, Erembodegem, Belgium), and CD45 (345808, clone 2D1; Becton Dickinson, Erembodegem, Belgium), and incubated in $100 \mu \mathrm{l} \alpha$-phenyltert-butylnitrone (PBN; phosphate-buffered saline; bovine serum albumin; and $\mathrm{NaN}_{3}$ ) with $2 \%$ human $\mathrm{AB}$ serum for $15 \mathrm{~min}$ at room temperature in the dark. Cells were washed with PBN twice, measured using a FACSARIA (Becton Dickinson, San Jose, CA), and evaluated using FACS Diva V6.1.2 software (Becton Dickinson, San Jose, CA).

\section{3 | Differentiation assays}

For adipogenic differentiation, BM-MSCs were cultured in Dulbecco's modified medium-low glucose (DMEM-LG) containing 10\% FBS-HI, $1 \mu \mathrm{M}$ dexamethasone (Invitrogen, Paisley, Scotland, UK), $60 \mu \mathrm{M}$ indomethacine Sigma, Saint Louis, MO), $500 \mu \mathrm{M}$ isobutylmethylxanthine (IBMX; Applichem, Germany), and $5 \mu \mathrm{g} / \mathrm{ml}$ Insulin (Sigma, Saint Louis, MO) for 21 days. Cells were fixed and stained with Oil. Oil Red O Red O(ORO) dye was extracted from MSCs using $2 \%$ Igepal in isopropanol for $15 \mathrm{~min}$ and assessed using an enzyme-linked immunosorbent assay (ELISA) plate reader (Tecan, Zürich, Switzerland) at $492 \mathrm{~nm}$. For osteogenic differentiation, BM-MSCs were grown in DMEM-LG containing 10\% FBS-HI, $100 \mathrm{nM}$ dexamethasone, $10 \mathrm{mM} \quad \beta$-glycerophosphate (Applichem, Germany), and $0.2 \mathrm{mM}$ L-ascorbic acid (Sigma, Saint Louis, MO) for 21 days. Cells were fixed and stained with Alizarin Red S (ARS) at pH 4.2 to confirm osteogenesis. The calcium content of wells was determined using the Quantichrom ${ }^{\mathrm{TM}}$ Ca assay kit (DICA-500; Bioassay Systems, Hayward, CA) and assessed using the ELISA plate reader at $690 \mathrm{~nm}$. To reduce ER stress, tauroursodeoxycholic acid sodium (TUDCA, $50 \mu \mathrm{M}$; Sigma, Saint Louis, MO) and 4-sodium phenylbutyrate (4-PBA, $1.0 \mathrm{mM}$; Sigma, Saint Louis, MO) were used.

\subsection{Real-time cell proliferation assessment (xCELLigence)}

Real-time proliferation of BM-MSCs was monitored using the xCELLigence RTCA DP instrument (Roche, Basel, Switzerland). Briefly, 
TABLE 1 Phenotyping of normal- and high-BMI donor BM-MSCs

\begin{tabular}{lllllllll} 
MSCS & CD29 & CD31 & CD44 & CD73 & CD90 & CD105 & CD166 & HLA-DR \\
\hline BMI $<25$ & $97.4 \pm 3.8$ & $0.4 \pm 0.4$ & $98.9 \pm 0.9$ & $97.4 \pm 4.8$ & $99.3 \pm 0.7$ & $98.5 \pm 2.0$ & $93.3 \pm 4.9$ & $6.0 \pm 1.4$ \\
BMI $>30$ & $86.8 \pm 4.7^{*}$ & $5.1 \pm 2.2^{*}$ & $77.2 \pm 3.5^{* *}$ & $90.3 \pm 1.2^{* *}$ & $89.2 \pm 4.8^{*}$ & $83.5 \pm 2.6^{* *}$ & $79.1 \pm 2.4^{*}$ & $7.9 \pm 4.4$ \\
\hline
\end{tabular}

${ }^{*} p<0.05$.

${ }^{* *} p<0.01$

2000 BM-MSCs from normal-BMI $(n=3)$ and high-BMI donors $(n=3)$ were added to $100 \mu \mathrm{l}$ of medium per well in triplicate in 96-well E-Plates (Roche, Basel, Switzerland). Cells were maintained in DMF10 medium and real-time proliferation was monitored for 10 consecutive days. Medium was changed after 5 days.

\subsection{Senescence assay}

BM-MSCs of donors with normal- and high-BMI were cultured in six-well dishes. Cells were fixed and stained according to the manufacturer's instructions using the senescence $\beta$-galactosidase staining kit (\#9860; Cell Signaling Technologies, Danvers, MA), where blue staining with $\beta$-galactosidase ( $\beta$-Gal) indicates senescent cells. Stained cells were imaged using bright-field microscopy (Olympus, Tokyo, Japan) and counted using the ImageJ software.

\section{6 | RT-PCR}

To assess gene expression, total RNAs were extracted from BM-MSCs using the RNA Protect Cell Reagent and RNeasy ${ }^{\circledR}$ Plus Mini Kit (Qiagen, Germany). RNA was converted into cDNA using the Quantitect Reverse Transcription Kit (Qiagen, Germany). Real-time polymerase chain reaction (RT-PCR) assays were performed with a LightCycler 480 II (Roche, Basel, Switzerland). Gene expressions of XBP-1 (Hs00231936_m1; Life Technologies), CHOP (HS00358796_g1; Life Technologies) and ATF4 (HS00909569_g1; Life Technologies), as indicators of ER stress; PPARG (ENST00000287820; Roche), LEP (ENSG00000174697; Roche) and SCD (ENSG00000099194; Roche) for adipogenic and ALPL (ENST00000374840; Roche) for osteogenic differentiation, respectively; and ACTB (HS01060665_g1, Life Technologies or ENST00000331789, Roche) as the housekeeping (a)

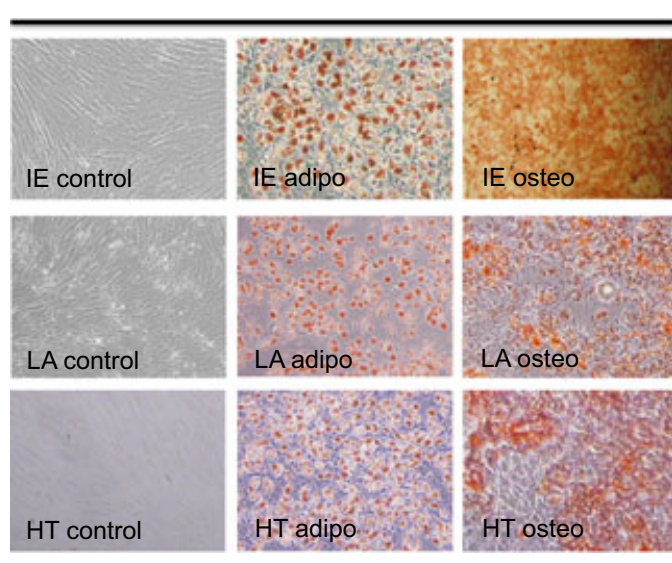

(b)

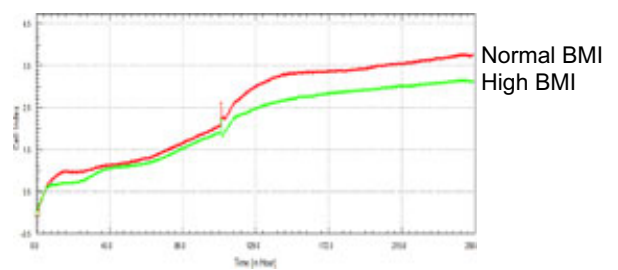

High BMI donor BM-MSCs

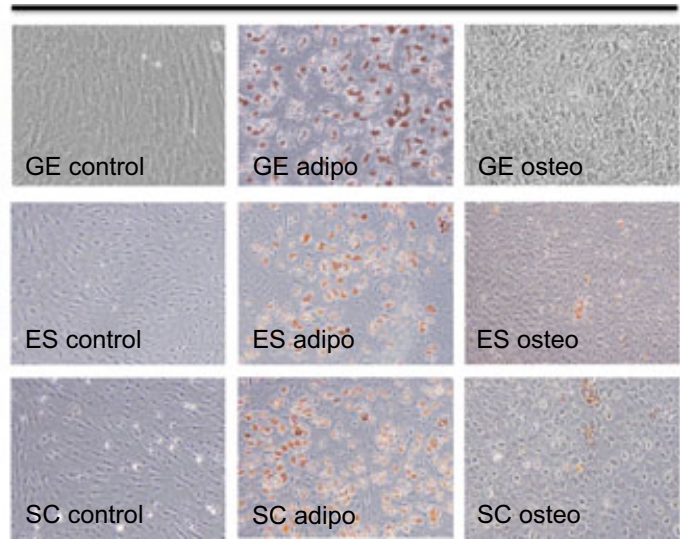

(c)

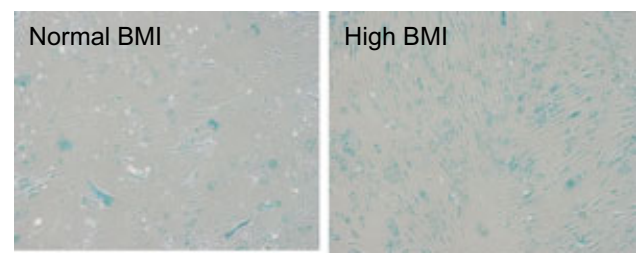

FIGURE 1 High-BMI BM-MSCs display reduced proliferation, decreased osteogenic differentiation, and increased senescence.

(a) Normal-BMI BM-MSCs $(n=3)$ and high-BMI BM-MSCs $(n=3)$ were differentiated for 21 days and stained with Oil Red O for adipogenic or Alizarin Red S for osteogenic cultures. Normal-BMI BM-MSCs (left) and high-BMI BM-MSCs (right) showed robust adipogenic differentiation, but osteogenic differentiation was markedly decreased in high-BMI BM-MSCs. (b) Real-time proliferation of normal-BMI BM-MSCs (red) and high-BMI BM-MSCs (green) was monitored for 10 consecutive days. Medium was changed after 5 days (peak at 100 hr). High-BMI BM-MSCs showed a decreased proliferation rate. (c) Representative picture of normal-BMI BM-MSCs (left) and high-BMI BM-MSCs (right). Blue staining indicates positivity for $\beta$-galactosidase. High-BMI BM-MSCs showed increased senescence. All photographs were taken using an inverted light microscope (Leica DMI6000 B, Leica Microsystems $\mathrm{GmbH}$, Germany) at $\times 10$ magnification. Abbreviations used in the pictures are initials of the individual donors. BMI, body mass index; BM, bone marrow; MSCs, mesenchymal stem cells [Color figure can be viewed at wileyonlinelibrary.com] 
gene were assessed in triplicate using SYBR Green I fluorescent labeled primers.

\section{7 | Statistical analysis}

Significant differences between the means of two groups were calculated using Student's t-test and variances between two groups were determined using F-tests in the GraphPad Prism software (www.graphpad.com/scientific-software/prism/).

$p<0.05$ were considered significantly different. The minimally required sample size for $90 \%$ power assuming a $5 \%$ significance level and a two-sided test was determined to be 5 . Correlations between BMI and osteogenic or adipogenic differentiation were calculated using Pearson's correlation in Microsoft excel for Mac 2011, version 14.7.3. Depending on the variables in the RT-PCR experiments, changes in expression were calculated by relative $\left(\Delta \Delta C_{t}\right)$ quantification.

\section{3 | RESULTS}

\subsection{High BMI affects the immunophenotype of BM-MSCs}

Morphologically, high-BMI BM-MSCs were more cubiform and larger than normal-BMI BM-MSCs. The immunophenotype of normal-BMI BM-MSCs was conform criteria, as defined by the International Society of Cellular Therapy (Dominici et al., 2006; Table 1). Surface expression of stromal adhesion molecules CD29 (Integrin $\beta 1$ ), CD44 (Hyaluronic acid receptor), and CD166 (ALCAM) was significantly lower on high-BMI BM-MSCs ( $p<0.05)$. The expression of MSC markers CD73, CD90, and CD105 was decreased on high-BMI
BM-MSCs $(p<0.05)$, but the epithelial marker CD31 was slightly increased on high-BMI BM-MSCs $(p<0.05)$.

\section{2 | High-BMI BM-MSCs are functionally impaired}

Osteogenic differentiation of high-BMI BM-MSCs was markedly impaired (Figure 1a). Calcium levels after 21 days of osteogenic differentiation were significantly lower $(p<0.02)$ in high-BMI BM-MSCs $(8.0 \pm 6.0 \mathrm{mg} / \mathrm{dl})$ in comparison with normal-BMI BM-MSCs $(14.2 \pm 4.8 \mathrm{mg} / \mathrm{dl})$. The ORO content of wells after 21 days of adipogenic differentiation of normal-BMI BM-MSCs was $0.70 \pm 0.58$ and that of high-BMI BM-MSCs was $0.28 \pm 0.13 \mathrm{mg} / \mathrm{ml}$ $(p=0.11)$. A negative correlation $(r=-0.46 ; p<0.04)$ was found between increasing $\mathrm{BMI}$ and osteogenic differentiation based on calcium phosphate levels in cultures after 21 days of differentiation. A similar negative correlation was not found between increasing BMI and adipogenic differentiation based on levels of Oil Red $\mathrm{O}$ after extraction from stained adipogenic cultures after 21 days of differentiation $(r=-0.26 ; p=0.35)$. Proliferation rates of high-BMI BM-MSCs were slower than those of normal-BMI BM-MSCs (Figure $1 \mathrm{~b}$ ), and the percentage of senescent ( $\beta$-galactosidase positive) cells in high-BMI BM-MSCs was increased (82.2 $\pm 15.1 \%$ ) in comparison with normal-BMI BM-MSCs (65.5 $\pm 29.8 \%$; Figure 1c).

\section{3 | High-BMI BM-MSCs display elevated gene expression of ATF4 and CHOP}

Expression levels of XBP1, ATF4, and CHOP mRNAs were assessed using quantitative RT-PCR on Days 1, 2, 4, and 7 of adipogenic and osteogenic differentiation of normal-BMI $(n=5)$ and high-BMI $(n=7)$ BM-MSCs (Figure 2). Baseline (before differentiation) mRNA levels of ATF4 and CHOP were, respectively, 1.9-fold $(p<0.01)$ and 2.8-fold (a)

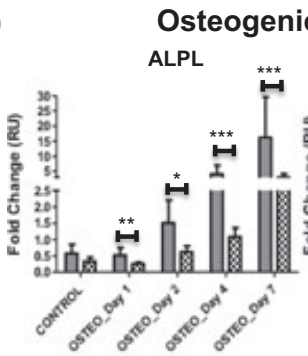

ATF4

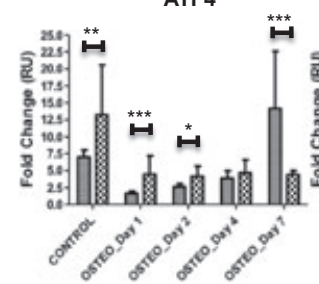

(b)

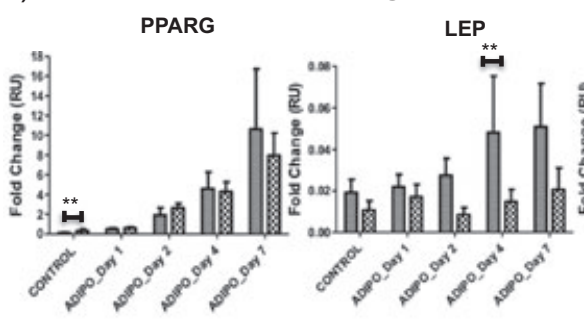

XBP1

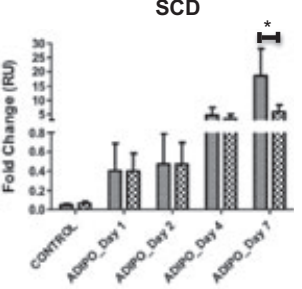

CHOP CHOP
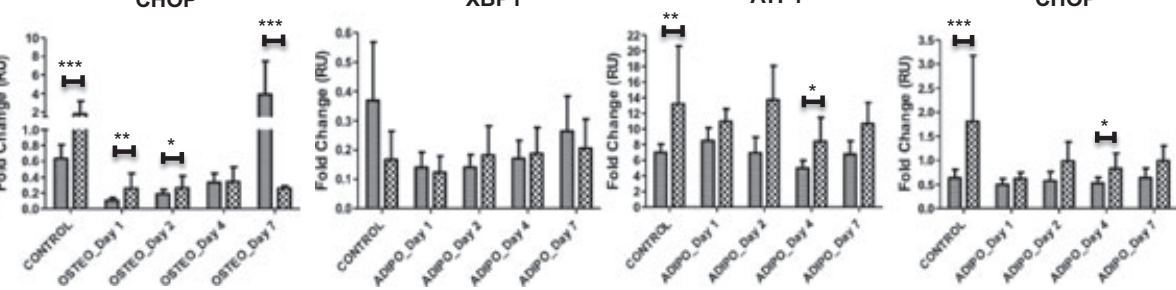

FIGURE 2 High-BMI BM-MSCs display impaired expression of osteogenic differentiation and ER stress-related genes and an inappropriate unfolded protein response. Gene expression of ALPL, as a marker of osteogenic differentiation, PPARG, LEP, and SCD, as markers of adipogenic differentiation, and XBP1, ATF4, and CHOP, as markers of ER stress under standard expansion culture conditions and during 7 days of adipogenic (a) and osteogenic differentiation, (b) in normal-BMI $(n=5)$ and high-BMI $(n=7)$ BM-MSCs. Data are given as mean \pm standard deviation. F-test was used to compare variances between two groups, ${ }^{*} p<0.05 ;{ }^{* *} p<0.01$; ${ }^{* * *} p<0.001$. BMI, body mass index; BM, bone marrow; MSCs, mesenchymal stem cells 
$(p<0.001)$ increased in high-BMI BM-MSCs in comparison with normal-BMI controls, whereas XBP1 levels were 2.2-fold lower in high-BMI donor MSCs (not statistically significant). Differences in the osteogenic differentiation of BM-MSCs were confirmed using ALPL gene expression (Figure 2a). Levels of ALPL transcripts increased highly (28.2-fold) during the 7 days of osteogenic differentiation of normal-BMI, but much less (9.2-fold) in high-BMI BM-MSCs. Differences in ALPL expression of high-BMI and normal-BMI donor MSCs were highly significant. In addition, high BMI was strongly correlated with a decrease in ALPL gene expression ( $r=-0.64$; $p<0,05)$. These data indicate an impaired osteogenic differentiation potential of high-BMI BM-MSCs, and confirm the previously observed lack of osteogenic changes in morphology, absence of ARS-positive staining, and decreased calcium-phosphate depositions. During osteogenic differentiation of normal-BMI BM-MSCs, gene expression of XBP1, ATF4, and CHOP decreased markedly at the start of differentiation, and then increased steadily over time and reached maximal expression levels on the last day. In high-BMI BM-MSCs, CHOP and ATF4 gene expression decreased markedly at Day 1 , but remained at low levels during the course of differentiation, whereas XBP1 levels remained largely unaffected. Differences in the expression of ER stress-related genes were highly significant between high$\mathrm{BMI}$ and normal-BMI BM-MSCs. These data indicate that high-BMI BM-MSCs have an impaired UPR during osteogenic differentiation, which may account for or contribute toward the decreased osteogenic differentiation potential of these cells. The adipogenic differentiation ability of BM-MSCs from normal-BMI and high-BMI donors was compared using the measurement of PPARG, LEP, and SCD gene expression (Figure $2 b$ ). Baseline gene expression levels of PPARG were increased in high-BMI BM-MSCs $(p<0.01)$, but LEP and SCD gene expression levels were not significantly different at baseline. During 7 days of adipogenic differentiation PPARG expression increased 91.0- and 24.7-fold; LEP expression increased 2.6- and 1.9-fold; and SCD expression increased 398.0 and 91.9 -fold in normal$\mathrm{BMI}$ and high-BMI BM-MSCs, respectively, indicating a relative defect in the adipogenic differentiation of high-BMI BM-MSCs as well. However, no significant correlation was found between high $\mathrm{BMI}$ and adipogenic differentiation based on PPARG $(r=-0.28 ; p=0.39)$, LEP $(r=-0.49 ; p=0.13)$, or $\operatorname{SCD}(r=-0.53 ; p=0.09)$ gene expression. During the adipogenic differentiation of normal-BMI BM-MSCs, XBP1 levels decreased in the beginning, and displayed a slow increase after the second day of differentiation, whereas XBP1 levels of high-BMI $B M-M S C s$ remained mainly unchanged. ATF4 gene expression levels in normal-BMI and high-BMI BM-MSCs showed minor fluctuations during differentiation, but levels of ATF4 transcripts were consistently higher in high-BMI BM-MSCs. CHOP gene expression in normal-BMI BM-MSCs was low at baseline and did not show change throughout adipogenic differentiation, whereas $\mathrm{CHOP}$ expression in high-BMI (a)

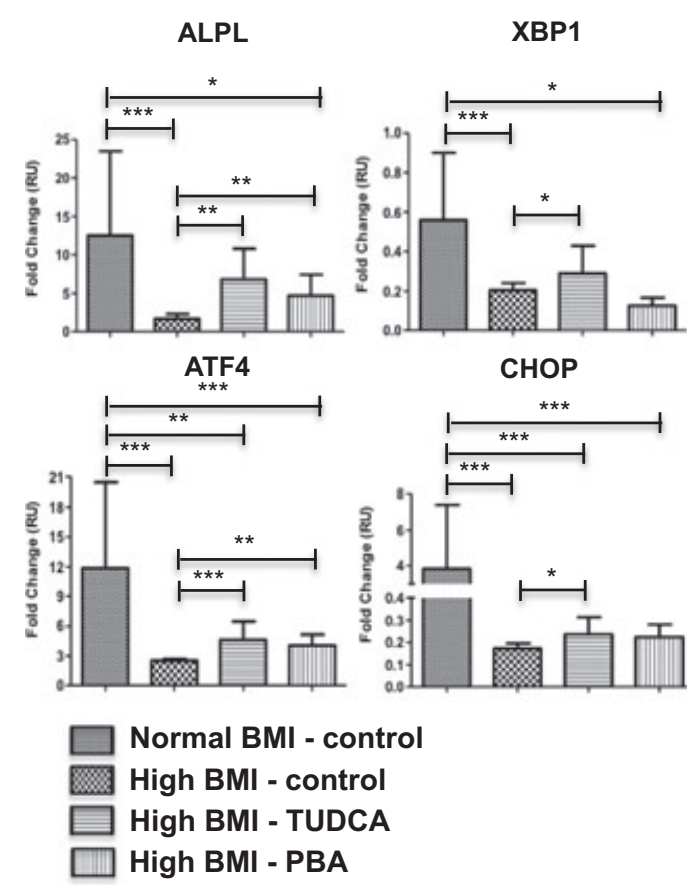

(b)

\section{Adipogenic differentiation}

PPARG

XBP1

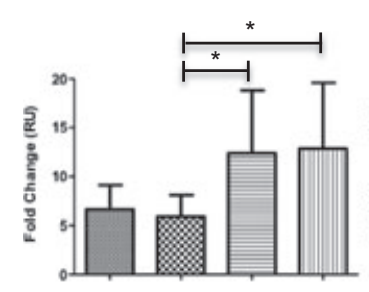

ATF4

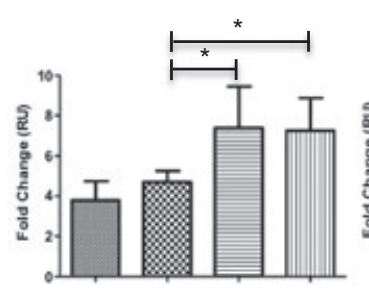

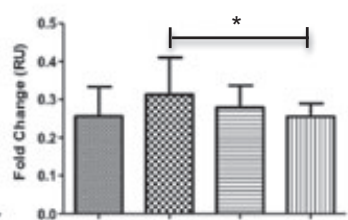

CHOP

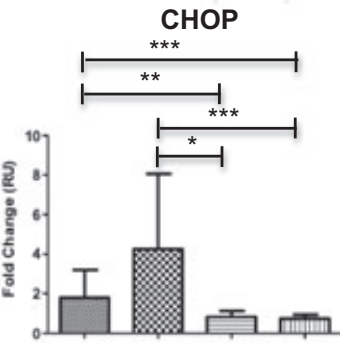

FIGURE 3 TUDCA and 4-PBA increase osteogenic and adipogenic differentiation of high-BMI BM-MSCs and lead to an improvement in the unfolded protein response. Gene expression of ALPL, XBP1, ATF4, and CHOP after 7 days of osteogenic (a) and adipogenic (b) differentiation of high-BMI BM-MSCs $(n=6)$ under control conditions (DMF10) and after treatment with TUDCA or 4-PBA. For comparison, data from normal-BMI BM-MSCs were added. Data are given as mean + standard deviation. Treatment with TUDCA and 4-PBA improves both osteogenic and adipogenic differentiation. The F-test was used to compare variances between two groups, ${ }^{*} p<0.05 ;{ }^{* *} p<0.01 ;{ }^{* * *} p<0.001$.

$\mathrm{BMI}$, body mass index; BM, bone marrow; MSCs, mesenchymal stem cells; 4-PBA, 4-phenylbutyrate; TUDCA, tauroursodeoxycholic acid; UPR, unfolded protein response 
BM-MSCs was increased at baseline, but rapidly decreased at Day 1 of adipogenic differentiation $(p<0.01)$.

\section{4 | TUDCA and 4-PBA improve osteogenic differentiation and normalize the UPR of high-BMI BM-MSCs}

Treatment of high-BMI BM-MSCs $(n=6)$ with TUDCA or 4-PBA resulted in a significant increase in ALPL gene expression (both conditions $p<0.01$ ) and partial recovery of osteogenic differentiation. After osteogenic differentiation for 7 days in the presence of TUDCA, gene expression of ATF4 ( $p<0.001)$, XBP1, and CHOP (both $p<0.05$ ) increased significantly (Figure $3 a$ ), resembling the gene expression pattern during UPR of normal-BMI BM-MSCs more closely, whereas osteogenic differentiation in the presence of 4-PBA showed only a significant effect on ATF4 expression $(p<0.01)$. After adipogenic differentiation in the presence of TUDCA or 4-PBA for 7 days, gene expression of PPARG and ATF4 was found to be significantly upregulated $(p<0.05)$, whereas CHOP was markedly downregulated compared with cultures without chaperones $(p<0.0001)$.

\section{4 | DISCUSSION}

BM-MSCs have rapidly gained importance for their use in regenerative medicine due to their relatively easy collection and expansion procedures, and their wide differentiation potential (Malgieri et al., 2010; Nombela-Arrieta et al., 2011; Odabas et al., 2014). Maintenance of differentiation capacity and stemness of BM-MSCs is crucial for their clinical application. With the steadily increasing rates of obesity $\left(\mathrm{BMI} \geq 30 \mathrm{~kg} / \mathrm{m}^{2}\right)$ in almost all populations in recent years (Turconi \& Hellas, 2007; WHO, 2000), the elucidation of the effects of obesity and its metabolism on stem cell functions has become equally more important. Obesity-related changes in metabolism affect the immune system, generate low-level chronic inflammation, cause impaired tissue healing, and most important of all, predispose to the development of many associated diseases, including Type 2 diabetes, cardiovascular disease, cancer, musculoskeletal diseases, and neurodegenerative diseases (Emanuela et al. 2012). Obesity negatively affects the function and maintenance of bone marrow hematopoietic stem cells and adipose stromal-derived stem cells (Ambrosi et al., 2017; Badimon \& Cubedo, 2017; Baptista et al., 2009; de Girolamo, Lucarelli, et al., 2013; De Girolamo, Stanco, et al., 2013), and has a negative impact on the differentiation of BMMSCs in mice (C. L. Wu, Diekman, et al., 2013 ).

An increase in mis- or unfolded proteins creates a stress condition in the ER called ER stress (Bravo et al., 2013). During physiological conditions, such as stem cell differentiation and proliferation, a certain level of ER stress may be required to compensate for increased cellular demands (Matsuzaki et al., 2015). However, pathophysiological conditions, such as obesity and diabetes, may induce ER stress as well (Ariyasu, Yoshida, \& Hasegawa, 2017; Januszyk et al., 2014; Pagliassotti, Kim, Estrada, Stewart, \& Gentile, 2016) and studies in mouse models have shown that both of these metabolic conditions may negatively affect stem cell behavior (Januszyk et al., 2014; D. Wu, Ren, Pae, Han, \& Meydani, 2013). The mechanisms through which obesity, in the absence of comorbidity, affects stem cell functions, and BM-MSCs in particular, are currently not fully understood, despite the presence of accumulating evidence indicating the importance of ER stress in the dysfunction of stem cells in obesity (Pagliassotti et al., 2016).

As a result of the increasing frequency of obesity in the general population, it is likely that a substantial fraction of future BM-MSC donors may be overweight or obese. This may have direct implications for their use, particularly in the field of regenerative medicine, where these cells could be potentially used for the treatment of orthopedic issues, such as critical-size bone fractures or cartilage defects. It is conceivable that BM-MSCs, ADSCs, or other stem cells, obtained from donors with advanced overweight, may display severe differentiation and proliferation defects, resulting in poor quality of the isolated stem cell product and decreased regenerative potential in vivo. Alleviation of ER stress, using a variety of ER stress inhibitors, including, but not restricted to, TUDCA and 4-PBA, could help improve the quality of the final stem cell product.

In this study, we found that baseline levels of ATF4 and CHOP were significantly increased in high-BMI BM-MSCs in comparison with normal-BMI controls. An increase in ATF4 and CHOP expression is typically seen after phosphorylation and activation of the PERK pathway and results in activation of the UPR. These data indicate that especially the PERK pathway appears to be activated in dealing with the negative results of obesity. Furthermore, the expressions of both ATF4 and $\mathrm{CHOP}$ should increase during normal osteogenic differentiation, but failed to do so in the high-BMI BM-MSC group. TUDCA has been shown to attenuate ER stress and prevent UPR dysfunction through prevention of Grp78/BiP upregulation, suppression of PERK and JNK phosphorylation, and prohibition of the expression of $\mathrm{CHOP}$ (Malo et al., 2010). In vitro, TUDCA was shown to enhance osteogenic differentiation of BM-MSCs (Cha et al., 2016; Han et al., 2013; Shi et al., 2017) and suppress the adipogenic differentiation of ADSCs (Cha et al., 2014). Here, we found that although TUDCA was very effective in increasing osteogenic differentiation, and to a lesser extent adipogenic differentiation, its effects were mainly visible as a normalization of the UPR. Treatment with TUDCA during osteogenic differentiation resulted in a significant increase in the expressions of both ATF4 and $\mathrm{CHOP}$ in highBMI BM-MSCs, more resembling gene expression levels in normal-BMI BM-MSCs. Similarly, treatment with TUDCA during adipogenic differentiation of high-BMI BM-MSCs resulted in an increase in ATF4, but with a concomitant decrease in $C H O P$, again resulting in expression levels more similar to normal-BMI BM-MSCs. 4-PBA modulates ER stress through the regulation of several ER stress-inducible UPR-related proteins, including Grp78/BiP, Grp94, C/EBP homologous protein, elF-2 $\alpha$, JNK, IRE-1 $\alpha$, PERK, and XBP-1 (Kim et al., 2012). Although 4-PBA was found to facilitate both osteogenic and adipogenic differentiation of BM-MSCs from high-BMI donors, the effects on ATF4 and CHOP were less robust. Since the administration of TUDCA and 4-PBA to high-BMI BM-MSCs resulted in an improvement in osteogenic differentiation and normalization of the UPR, and since 
4-PBA and TUDCA modulate different UPR pathways, it is conceivable that optimized protocols, including both chaperones together or consecutively, might completely repair or at least further improve osteogenic differentiation. In addition, the fact that treatment with TUDCA and 4-PBA, which are known to modulate ER stress through suppression of the UPR, appear to partially correct the osteogenic differentiation of high-BMI BM-MSCs by increasing (normalizing), rather than decreasing, the expression of ATF4 and CHOP, suggests that TUDCA and 4-PBA may also act through other pathways.

In this study, we hypothesized that ER stress and the UPR might play a pivotal role in coping with cellular stress, and maintenance of the function and stemness of high-BMI BM-MSCs. We compared and characterized BM-MSCs from normal-BMI (<25) and high-BMI (>30) donors, and evaluated the levels of ER stress during expansion and differentiation. High-BMI BM-MSCs displayed a decreased proliferation rate, increased senescence, and increased levels of ER stress-related genes ATF4 and CHOP. Osteogenic differentiation of high-BMI BM-MSCs was severely disturbed, with almost no morphological signs of differentiation, low-level gene expression of ALPL, and a severely disturbed UPR. In contrast, high-BMI BM-MSCs showed nearly normal adipocytic differentiation and close to normal expression of PPARG, LEP, and SCD. Administration of TUDCA and 4-PBA resulted in improved osteogenic differentiation, increased adipogenic differentiation, and normalization of the UPR. These results are important for the development of stem cell therapies for and from patients with high BMI. Further understanding of the effects of obesity on stem cell behavior may enable optimization of stem cell products for therapeutic use and result in the discovery of new treatment strategies and targets.

\section{ACKNOWLEDGMENTS}

The authors would like to thank clinical research nurse Hacer Cüzdancı for her help with collection of the samples and Prof. Dr. Emin Kansu for critically reviewing the manuscript. The research was supported by the EU 7th Framework Program (no. CELL-PID 261387) and The Turkish Society of Hematology (no. 2015/03).

\section{CONFLICTS OF INTEREST}

The authors declare that there is no conflicts of interest.

\section{ORCID}

Fatima Aerts-Kaya (D) http://orcid.org/0000-0002-9583-8572

\section{REFERENCES}

Adler, B. J., Kaushansky, K., \& Rubin, C. T. (2014). Obesity-driven disruption of haematopoiesis and the bone marrow niche. Nature Reviews Endocrinology, 10(12), 737-748.
Ambrosi, T. H., Scialdone, A., Graja, A., Gohlke, S., Jank, A. M., Bocian, C., ... Schulz, T. J. (2017). Adipocyte accumulation in the bone marrow during obesity and aging impairs stem cell-based hematopoietic and bone regeneration. Cell Stem Cell, 20(6), 771-784. e776.

Ariyasu, D., Yoshida, H., \& Hasegawa, Y. (2017). Endoplasmic reticulum (ER) stress and endocrine disorders. International Journal of Molecular Sciences, 18(2), 382.

Badimon, L., \& Cubedo, J. (2017). Adipose tissue depots and inflammation: Effects on plasticity and resident mesenchymal stem cell function. Cardiovascular Research, 113, 1064-1073.

Baptista, L. S., da Silva, K. R., da Pedrosa, C. S., Claudio-da-Silva, C., Carneiro, J. R., Aniceto, M., ... Borojevic, R. (2009). Adipose tissue of control and ex-obese patients exhibit differences in blood vessel content and resident mesenchymal stem cell population. Obesity Surgery, 19(9), 1304-1312.

Basseri, S., Lhotak, S., Sharma, A. M., \& Austin, R. C. (2009). The chemical chaperone 4-phenylbutyrate inhibits adipogenesis by modulating the unfolded protein response. Journal of Lipid Research, 50(12), 2486-2501.

Bravo, R., Parra, V., Gatica, D., Rodriguez, A. E., Torrealba, N., Paredes, F., ... Lavandero, S. (2013). Endoplasmic reticulum and the unfolded protein response: Dynamics and metabolic integration. International Review of Cell and Molecular Biology, 301, 215-290.

Cha, B. H., Jung, M. J., Moon, B. K., Kim, J. S., Ma, Y., Arai, Y., ... Lee, S. H. (2016). Administration of tauroursodeoxycholic acid enhances osteogenic differentiation of bone marrow-derived mesenchymal stem cells and bone regeneration. Bone, 83, 73-81.

Cha, B. H., Kim, J. S., Ahn, J. C., Kim, H. C., Kim, B. S., Han, D. K., ... Lee, S. H. (2014). The role of tauroursodeoxycholic acid on adipogenesis of human adipose-derived stem cells by modulation of ER stress. Biomaterials, 35(9), 2851-2858.

Dominici, M., Le Blanc, K., Mueller, I., Slaper-Cortenbach, I., Marini, F., Krause, D., ... Horwitz, E. (2006). Minimal criteria for defining multipotent mesenchymal stromal cells. Cytotherapy, 8(4), 315-317.

Emanuela, F., Grazia, M., Marco de, R., Maria Paola, L., Giorgio, F., \& Marco, B. (2012). Inflammation as a link between obesity and metabolic syndrome. Journal of Nutrition and Metabolism, 2012, 476380-476387.

Emmons, R., Niemiro, G. M., \& De Lisio, M. (2017). Hematopoiesis with obesity and exercise: Role of the bone marrow niche. Exercise Immunology Review, 23, 82-95.

de Girolamo, L., Lucarelli, E., Alessandri, G., Avanzini, M. A., Bernardo, M. E., Biagi, E., ... Stem Cell, G. (2013). Mesenchymal stem/stromal cells: A new "cells as drugs" paradigm. Efficacy and critical aspects in cell therapy. Current Pharmaceutical Design, 19(13), 2459-2473.

De Girolamo, L., Stanco, D., Salvatori, L., Coroniti, G., Arrigoni, E., Silecchia, G., ... Brini, A. T. (2013). Stemness and osteogenic and adipogenic potential are differently impaired in subcutaneous and visceral adipose derived stem cells (ASCs) isolated from obese donors. International Journal of Immunopathology and Pharmacology, 26(1 Suppl), 11-21.

Han, J., Murthy, R., Wood, B., Song, B., Wang, S., Sun, B., ... Kaufman, R. J. (2013). ER stress signalling through elF2alpha and CHOP, but not IRE1alpha, attenuates adipogenesis in mice. Diabetologia, 56(4), 911-924.

Januszyk, M., Sorkin, M., Glotzbach, J. P., Vial, I. N., Maan, Z. N., Rennert, R. C., ... Gurtner, G. C. (2014). Diabetes irreversibly depletes bone marrow-derived mesenchymal progenitor cell subpopulations. Diabetes, 63(9), 3047-3056.

Kim, D. S., Li, B., Rhew, K. Y., Oh, H. W., Lim, H. D., Lee, W., ... Kim, H. R. (2012). The regulatory mechanism of 4-phenylbutyric acid against ER stress-induced autophagy in human gingival fibroblasts. Archives of Pharmacal Research, 35(7), 1269-1278.

Malgieri, A., Kantzari, E., Patrizi, M. P., \& Gambardella, S. (2010). Bone marrow and umbilical cord blood human mesenchymal stem cells: 
State of the art. International Journal of Clinical and Experimental Medicine, 3(4), 248-269.

Malo, A., Kruger, B., Seyhun, E., Schafer, C., Hoffmann, R. T., Goke, B., \& Kubisch, C. H. (2010). Tauroursodeoxycholic acid reduces endoplasmic reticulum stress, trypsin activation, and acinar cell apoptosis while increasing secretion in rat pancreatic acini. AGastrointestinal and Liver Physiology, 299(4), G877-G886.

Matsuzaki, S., Hiratsuka, T., Taniguchi, M., Shingaki, K., Kubo, T., Kiya, K., ... Katayama, T. (2015). Physiological ER stress mediates the differentiation of fibroblasts. PLoS One, 10(4), e0123578.

Mihaylova, M. M., Sabatini, D. M., \& Yilmaz, O. H. (2014). Dietary and metabolic control of stem cell function in physiology and cancer. Cell Stem Cell, 14(3), 292-305.

Nombela-Arrieta, C., Ritz, J., \& Silberstein, L. E. (2011). The elusive nature and function of mesenchymal stem cells. Nature Reviews Molecular Cell Biology, 12(2), 126-131.

Odabas, S., Elcin, A. E., \& Elcin, Y. M. (2014). Isolation and characterization of mesenchymal stem cells. Methods in Molecular Biology, 1109, 47-63.

Pagliassotti, M. J., Kim, P. Y., Estrada, A. L., Stewart, C. M., \& Gentile, C. L. (2016). Endoplasmic reticulum stress in obesity and obesity-related disorders: An expanded view. Metabolism: Clinical and Experimental, 65(9), 1238-1246.

Park, S. W., \& Ozcan, U. (2013). Potential for therapeutic manipulation of the UPR in disease. Seminars in Immunopathology, 35(3), 351-373.

Shi, M., Song, W., Han, T., Chang, B., Li, G., Jin, J., \& Zhang, Y. (2017). Role of the unfolded protein response in topography-induced osteogenic differentiation in rat bone marrow mesenchymal stem cells. Acta Biomaterialia, 54, 175-185.

Strioga, M., Viswanathan, S., Darinskas, A., Slaby, O., \& Michalek, J. (2012). Same or not the same? Comparison of adipose tissue-derived versus bone marrow-derived mesenchymal stem and stromal cells. Stem Cells and Development, 21(14), 2724-2752.

Turconi, G., \& Hellas, C. (2007). Epidemiology of obesity. Obesity, epidemiology, pathophysiology and prevention. In Bell, J. S. (Ed.), Obesity. USA: Taylor \& Francis Group.

WHO. 2000. Obesity: Preventing and managing the global epidemic (256p). Report of a WHO consultation on obesity. Geneva, Switserland.

Wu, C. L., Diekman, B. O., Jain, D., \& Guilak, F. (2013). Diet-induced obesity alters the differentiation potential of stem cells isolated from bone marrow, adipose tissue and infrapatellar fat pad: The effects of free fatty acids. International Journal of Obesity (2005), 37(8), 1079-1087.

Wu, D., Ren, Z., Pae, M., Han, S. N., \& Meydani, S. N. (2013). Diet-induced obesity has a differential effect on adipose tissue and macrophage inflammatory responses of young and old mice. Biofactors, 39(3), 326-333.

Wu, J., \& Kaufman, R. J. (2006). From acute ER stress to physiological roles of the unfolded protein response. Cell Death and Differentiation, 13(3), 374-384.

How to cite this article: Ulum B, Teker HT, Sarikaya A, et al. Bone marrow mesenchymal stem cell donors with a high body mass index display elevated endoplasmic reticulum stress and are functionally impaired. J Cell Physiol. 2018;233:8429-8436. https://doi.org/10.1002/jcp.26804 